\title{
Water Content Analysis of Sludge using NMR Relaxation Data and Independent Component Analysis
}

\author{
Mika Liukkonen $^{1} \quad$ Ekaterina Nikolskaya $^{2} \quad$ Jukka Selin $^{2} \quad$ Yrjö Hiltunen ${ }^{2}$ \\ ${ }^{1}$ Department of Environmental and Biological Sciences, University of Eastern Finland, Finland, \\ mika.liukkonen@uef.fi \\ Fiber Laboratory, South-Eastern Finland University of Applied Sciences, Finland, \\ \{ekaterina.nikolskaya, jukka.selin, yrjo.hiltunen\} @xamk.fi
}

\begin{abstract}
In wastewater treatment, the dewatering of sludge is one of the most important steps, because it affects largely in both the process economics and the costs of sludge disposal. To optimize the dewatering processes, it would be beneficial to be aware of the different water types present in the sludge. In addition to free water, generally there are also mechanically, physically and chemically bound water within the sludge. All these water types behave differently when the sludge is dried, and they all require a different amount of energy when being removed. In this study, the Independent Component Analysis (ICA) method has been applied to an analysis of NMR (Nuclear Magnetic Resonance) relaxation data obtained from the measurement of wastewater sludge samples with a known moisture content. The results strongly suggest that the ICA method can be used for determining the amount of different water types within the wastewater sludge without a priori knowledge on their shares.
\end{abstract}

Keywords: independent component analysis, water content, nuclear magnetic resonance, sludge, relaxation decay

\section{Introduction}

Sludge is a semi-solid by-product remaining after wastewater treatment, industrial or refining processes. It is a separated solid suspended in a liquid, characteristically comprising large quantities of interstitial water between its solid particles (Global Water Community, 2015). This material can be dried to reduce its volume and to remove most of the moisture content of the solids within the sludge (Global Water Community, 2015). In wastewater treatment, the dewatering of sludge is one of the most important steps, because it affects largely both the process economics and the costs of sludge disposal.

It is suggested by several authors that the moisture in activated sludge can be classified to the following four categories (Kopp \& Dichtl, 2000; Vesilind 1994; Tsang \& Vesiling, 1990; Vesilind \& Hsu, 1997; Smith \& Vesiling, 1995):
- Free water: water which is not bound to the particles, including void water not affected by the capillary force.

- Interstitial water: water bound by capillary forces inside crevices and interstitial spaces of flocs.

- Surface water: water bound to the surface of solid particles by adhesive forces.

- Bound intracellular water.

This is a widely accepted classification and can be used as the reference in determining the main water types of sludge.

Another classification of water types in sludge is to divide it in three groups, i.e. 1) free water, 2) mechanically bound water, and 3) physically or chemically bound water. The free water in sludge can be easily removed by mechanical means, whereas the bound water is held firmly within the floc, bound to the sludge or trapped between the sludge particles, and thus cannot be easily removed (Jin et al., 2004). The bound water can be further divided into chemically or physically bound water which is removable only by thermal drying, and mechanically bound water which is bound by weaker capillary forces (Colin \& Gazbar, 1995).

In summary, it has to be emphasized that determining the water types is not straightforward, and based on the literature it is difficult to reach an unambiguous interpretation on the distribution of water within activated sludge (Vaxelaire \& Cézac, 2004). Furthermore, there seem to be no studies concentrating on the analysis of water types in sludge without a priori knowledge of the shares of different water types.

Time domain nuclear magnetic resonance method (TD- NMR) is also becoming highly attractive for industrial applications due to relatively low price, mobility, easy operating, and simple sample preparation procedure. The most successful applications of TDNMR confirmed by international standards are solid fat content determination in food and water (ISO 8292) and oil content in oilseeds (ISO 10565). They are based on the difference of NMR parameters of water and lipids and a low exchange degree between these two fractions. 
A possibility to use the same principle for analysis of lipid content in microalgae (Gao et al., 2008), for analysis of oil content of olive mill wastes and municipal wastewater sludge (Willson et al., 2010) was demonstrated. Effects of flocculation on the bound water in sludge as measured by the NMR spectroscopy has been studied by Carberry and Prestowitz (1985).

Moreover, the international standard for hydrogen content determination in aviation fuels (ASTM D7171 $05,2011)$ has been developed recently. Metal ions, particularly paramagnetic ions, can also change significantly relaxation times in water and biological samples (Yilmaz et al., 1999; Grunin et al., 2013) which can be applicable when controlling wastewater treatment. Time domain NMR data have also been used in analyzing the water contents of wood and peat based fuels (Nikolskaya et al., 2011) and monitoring the precipitation of metals in mine waters (Nikolskaya et al., 2015).

Independent component analysis (ICA) is a statistical method that has been successfully applied to a variety of problems in signal processing (Hyvärinen et al., 2001). For example, the method has been applied to a variety of problems in several fields such as brain imaging (Pulkkinen et al., 2005; Calhoun et al., 2002), vision research (Zhang \& Mei, 2003; Ameen \& Szu, 1999), telecommunications (Ristaniemi \& Joutsensalo, 1999) and financial research (Kiviluoto \& Oja, 1998; Back \& Weigend, 1997). ICA is a method for extracting underlying, fundamental factors or components from multivariate data. It is designed so that it searches for components that are both statistically independent and non-Gaussian (Hyvärinen et al., 2001), which makes it a distinguished method among the other techniques.

The complexity of spectral information can be approached by assuming that the obtained spectra are statistically independent. Principal component analysis (PCA) is the standard approach to analyze spectral data (Hyvärinen et al., 2001). PCA is based on second-order statistics, which is applicable in the analysis of Gaussian distributed data. However, spectral data can comprise interesting information having a non-Gaussian distribution that can potentially be analyzed with ICA.

In the present study, the ICA method has been applied to an analysis of NMR relaxation data obtained from the measurement of wastewater sludge samples with a known moisture content.

\section{Materials and methods}

\subsection{NMR measurements}

The seven sludge samples (See Table 1) were obtained from an industrial waste water treatment plant. The samples were gathered after the dewatering stage of the process. The water contents of samples were measured using the standard oven drying method.
Relaxation times measurements were done using a mobile NMR device with a $1 \mathrm{H}$ resonance frequency of 25.7 MHz (Resonance Systems Ltd). The device has been modified for online measurements in industrial conditions. The permanent magnet of $0.6 \mathrm{~T}$ has dimension of 140x190x150 mm weighting $19 \mathrm{~kg}$. The diameter of sensor hole was $10 \mathrm{~mm}$. CPMG (CarrParcell-Meiboom-Gill) pulse sequence for spin-spin relaxation time $\mathrm{T} 2$ measurements was used.

Table 1. Description of sludge samples

\begin{tabular}{|c|c|}
\hline Sample ID & Water content [\%] \\
\hline Sample 1 & 54 \\
\hline Sample 2 & 68 \\
\hline Sample 3 & 75 \\
\hline Sample 4 & 79 \\
\hline Sample 5 & 83 \\
\hline Sample 6 & 85 \\
\hline Sample 7 & 89 \\
\hline
\end{tabular}

\subsection{Independent Component Analysis}

It is assumed here that there are $n$ observed signals (i.e., types of water), $\mathrm{WS}_{1}, \mathrm{WS}_{2}, \ldots, \mathrm{WS}_{n}$ in the data, which are linear combinations of $m$ independent components, $\mathrm{IC}_{l}, \mathrm{IC}_{2}, \ldots, \mathrm{IC}_{m}$. The equation for $\mathrm{IC}_{i}$ can be written as:

$$
\begin{gathered}
W S_{i}=a_{i 1} I C_{1}+a_{i 2} I C_{2}+\cdots+a_{i m} I C_{m} \\
=\sum_{j=1}^{m} a_{i j} I C_{j}
\end{gathered}
$$

where $i=1,2, \ldots, n$ and the $a_{i j}$ are real coefficients (contributions of ICs). The independent components, $\mathrm{IC}_{j}$, and also the corresponding coefficients, $a_{i j}$, are unknown.

The statistical model in Eq. (1) is called the independent component analysis model (Hyvärinen et al., 2001). The ICA model is a generative model that describes how the observed data are generated by a process of mixing the components $\mathrm{IC}_{i}$. Both $\mathrm{IC}_{i}$ and $a_{i j}$ need to be estimated using the observed data. The starting point for ICA is the assumption that the components $\mathrm{IC}_{i}$ are statistically independent, which can be concluded from non-gaussianity (Hyvärinen et al., 2001). Here, a fixed-point algorithm (Fast-ICA) was used as an implementation of ICA (Hyvärinen et al., 2001). The analysis was performed using the Fast-ICA toolbox under the Matlab software platform (Mathworks, Natick, MA, USA).

After the analysis, the relative shares of each component can be calculated using the following formula:

$$
a_{i j, r e l}=\frac{a_{i j}}{\sum_{j=1}^{m} a_{i j}} \times 100
$$




\section{Results}

The original NMR measurement signals can be seen in Fig. 1. It can be seen that there is a clear dependency between the amplitude of the signal and the moisture content of sludge samples. The gained NMR relaxation data were then analyzed by the ICA method based on the Hyvärinen's fixed-point algorithm (Hyvärinen et al., 2001). Several numbers of ICs were tested, and three ICs were eventually used, because this setting was found to yield the most consistent and stable results. The three independent components (IC) can be seen in Fig. 2. It can be seen that all three ICs have their own, independent behavior.

According to theory, the share of bound water from the total amount of water remains stable in the sludge when the water content is increased from 0 on to a certain point (See Fig. 3, above). After this point, other types of water start to accumulate. When moisture content is $100 \%$, all water is considered to be in a free form, but when the sludge is dried, the share of free water decreases dramatically, and the share of bound water increases. The share of the so called interstitial water (bound by weaker capillary forces) reaches its highest value at around 70-90\% moisture content.

In Fig. 3 (below), the calculated relative shares of different water types as a function of the total water content of the samples can be seen. It can be seen that the measured and analyzed values roughly follow the theoretic values and thus support them.

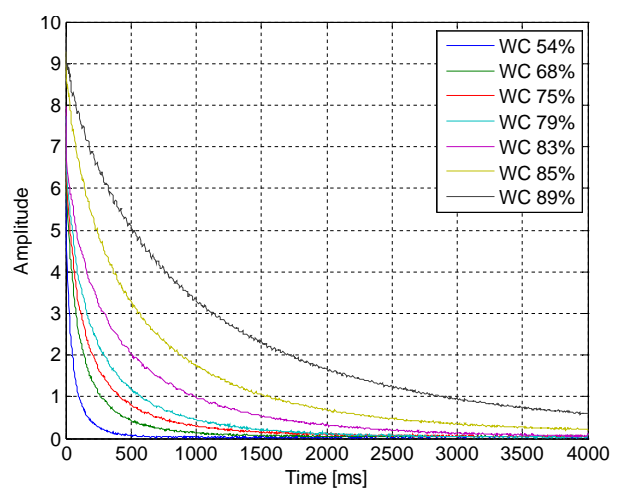

Figure 1. The observed NMR measurement signals (WS) from the 7 samples. $\mathrm{WC}=$ the water content of sample.

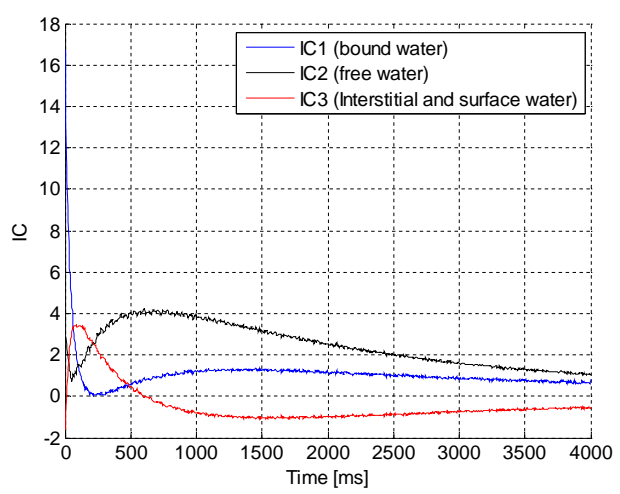

Figure 2. The three independent components (IC) computed from the NMR relaxation data.
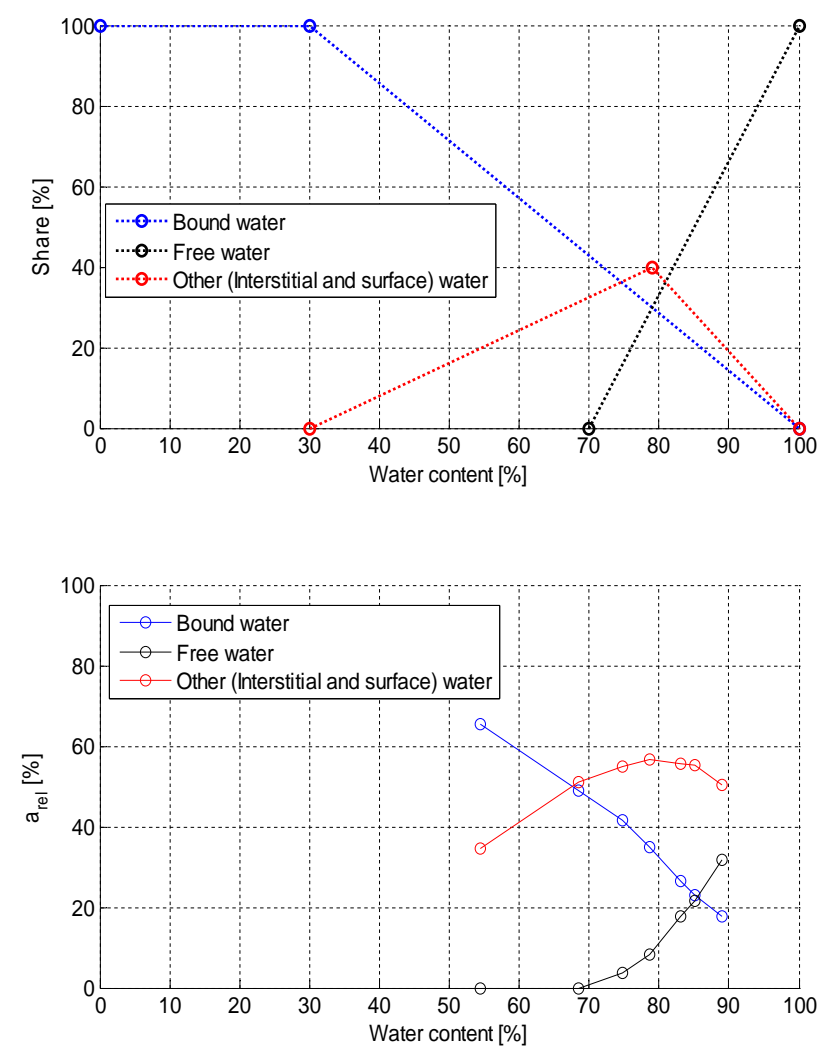

Figure 3. Theoretic (above) and calculated (below) relative shares of different water types as a function of the total water content of the samples.

\section{Discussion}

Measurement of the different water types in sludge is an exceptionally challenging problem, and to our knowledge this has not been tried before. In this respect, the results are extremely promising.

ICA is a universal statistical technique in which observed data are linearly transformed into components that are maximally independent from each other. A key issue in using the ICA method is to decide the number of ICs to be estimated. For the data set used, only the physically meaningful components were chosen. Our results support the use of three independent components in this case. This suggests that there are three types of signals in this data.

There is no universal truth on how many water types are present in sludge. The four water types including free, interstitial, surface and bound water, are widely accepted, but also other viewpoints exist. In this particular case three independent components could be most easily extracted from the NMR relaxation data. This suggests that there are three signals that are maximally and statistically independent when it comes to their spectra, but this does not mean that there could be more water types present as well.

Based on the results it seems that the combination of time-domain NMR and ICA can be used for determining the amount of different water types within the 
wastewater sludge. It is also beneficial that the ICA method does not require a priori knowledge on the water types and their shares in the sludge. This makes it very specific and a promising approach to optimize the dewatering processes of sludge.

\section{Conclusions}

Based on the results it can be concluded that incorporating ICA into data analysis allows for decomposition of independent, systematically occurring patterns in NMR relaxation data. This new information can be used for guiding further study and may lead to a way of extracting the shares of different water types in wastewater sludge. This would help in making the sludge dewatering more economical and in reducing the costs of sludge disposal.

\section{Acknowledgements}

This research is a part of the InDiGO! (Intelligent Software and Service Concept of the Industrial Internet) project, which is funded by the Finnish Funding Agency for Technology and Innovation (TEKES). Furthermore, Savonlinna Smart Demonstrations (SMD) project (funded by the South Savo Regional Council and the European Regional Development Fund, ERDF) is acknowledged.

\section{References}

Global Water Community. Sludge Drying Overview Treatment Methods and Applications. 2015. Available via: http://www.iwawaterwiki.org/xwiki/bin/view/Articles/Slu dgeDryingOverview-TreatmentMethodsandApplications

J. Kopp and N. Dichtl. The Influence of Free Water Content on Sewage Sludge Dewatering. In: Chemical Water and Wastewater Treatment VI, H.H. Hahn, E. Hoffmann and H. Ødegaard, Eds. Berlin Heidelberg New York: SpringerVerlag, 2000, pages 347-356.

P.A.Vesilind. The role of water in sludge dewatering. Water Environ Res, 66(1):4-11, 1994.

K.R. Tsang and P.A. Vesilind. Moisture distribution in sludges. Water Sci Technol, 22(12):135-142, 1990.

P.A. Vesilind and C.C. Hsu. Limits of sludge dewaterability. Water Sci Technol, 36(11):87-91, 1997.

J.K. Smith and P.A. Vesilind. Dilatometric measurement of bound water in wastewater sludge. Water Res, 29(12):26212626, 1995

B. Jin, B.-M. Wilén and P. Lant. Impacts of morphological, physical and chemical properties of sludge flocs on dewaterability of activated sludge. Chemical Engineering Journal, 98:115-126, 2004

F. Colin and S. Gazbar. Distribution of water in sludges in relation to their mechanical dewatering. Water Res, 29:2000-2005, 1995.

J. Vaxelaire and P. Cézac. Moisture distribution in activated sludges: a review. Water Res, 38:2215-2230, 2004.

ISO 8292: 2008(en) Animal and vegetable fats and oils Determination of solid fat content by pulsed NMR. 2008.

ISO 10565: 1998 Oilseeds - Simultaneous determination of oil and water contents - Method using pulsed nuclear magnetic resonance spectrometry. 1998.

C. Gao, W. Xiong, Y. Zhang, W. Yuan and Q. Wu. Rapid quantitation of lipid in microalgae by time-domain nuclear magnetic resonance. Journal of Microbiological Methods, 75:437-440, 2008.

R.M. Willson, Z. Wiesman and A. Brenner. Analyzing alternative bio-waste feedstocks for potential biodiesel production using time domain (TD)-NMR. Waste Management, 30:1881-1888, 2010.

J. Bower Carberry and R.A. Prestowitz. Flocculation Effects on Bound Water in Sludges as Measured by Nuclear Magnetic Resonance Spectroscopy. Applied and Environmental Microbiology, 49(2):365-369, 1985.

ASTM D7171 - 05: 2011 Standard Test Method for Hydrogen Content of Middle Distillate Petroleum Products by LowResolution Pulsed Nuclear Magnetic Resonance Spectroscopy. 2011.

A. Yilmaz, M. Yurdakoc and B. Isik. Influence of transition metal ions on NMR proton T1 relaxation times of serum, blood, and red cells. Biological Trace Element Research, 67:187-193, 1999.

L. Grunin, E. Nikolskaya and J. Edwards. The use of 1HNMR Relaxation Times of Water Adsorbed on Soils to Monitor Environment Pollution. Air, Soil and Water Research, 6:115-119, 2013.

E. Nikolskaya, M. Liukkonen, R.A. Kauppinen, L. Grunin and Y. Hiltunen. Water contents of Wood and Peat Based Fuels by Analyzing Time Domain NMR data. In: E. Dahlquist, Ed., Proc. the 52nd International Conference of Scandinavian Simulation Society, SIMS 2011, paper 6, 2011.

E. Nikolskaya, M. Liukkonen, J. Kankkunen and Y. Hiltunen. A non-fouling online method for monitoring precipitation of metal ions in mine waters. IFAC Proceedings Volumes (IFAC papers online), 48(17):98-101, 2015.

A. Hyvärinen, J. Karhunen and E. Oja. Independent Component Analysis. John Wiley \& Sons, New York. 2001.

J. Pulkkinen, A. Häkkinen, N. Lundbom, A. Paetau, R. Kauppinen and Y. Hiltunen. Independent component analysis to proton spectroscopic imaging data of human brain tumours. European Journal of Radiology, 56(2):160164, 2005.

V.D. Calhoun, T. Adali, G.D. Pearlson, P.C.M. van Zijl and J.J. Pekar. Independent component analysis of fMRI data in the complex domain. Magnetic Resonance in Medicine, 48:180-192, 2002.

L. Zhang and J. Mei. Shaping up simple cell's receptive field of animal vision by ICA and its application in navigation system. Neural Networks, 16(5-6):609-615, 2003.

M. Ameen and H. Szu. Early vision image analyses using ICA in unsupervised learning ANN. In: Proc. International Joint Conference on Neural Networks (IJCNN '99), vol. 2, pages 1022-1027, 1999.

T. Ristaniemi and J. Joutsensalo. On the performance of blind source separation in CDMA downlink. In: Proc. Int. Workshop on Independent Component Analysis and Signal Separation (ICA'99), pages 437-441, 1999.

K. Kiviluoto and E. Oja. Independent component analysis for parallel financial time series. In: Proc. Int.Conf. on Neural Information Processing (ICONIP'98), vol. 2, pages 895898, 1998.

A. D. Back and A. S. Weigend. A first application of independent component analysis to extracting structure from stock returns. Int. J. on Neural Systems, 8(4):473-484, 1997. 